\section{Further improvement of vertical jumping performance of volleyball players through targeted plyometric training ${ }^{1}$}

Hedef gösterilerek yapılan pliometrik çalışmalar voleybolcuların dikey sıçrama performansını daha fazla geliştiriyor

\author{
Mehmet Yildiz ${ }^{2}$ \\ Oğuzhan Engin ${ }^{3}$
}

\begin{abstract}
Background and Aim: The purpose of this study was to investigate the effect of jumping exercises with an overhead target on vertical jump performance of volleyball players. Method: Eighteen male volleyball players (age $21.3 \pm 1.8$ years, height $183.8 \pm 10.0 \mathrm{~cm}$, weight $78.44 \pm 12.0 \mathrm{~kg}$ ) participated in the study. The participants were divided into two groups randomly. Before the study, as a pretest, jumping heights of all participants were measured by the Sargent method. Then, three days a week, for a total of four weeks, the control group performed 10 vertical jump exercises without targeting, while the study group performed 10 jumps with 10\% more of the pretest result after the standard volleyball training. The target of a participant who reached the target three times in one session was increased by $10 \%$. As a final test, maximal jump heights of both groups were measured again with the Sargent method. A 2X2 repeated measures analysis of variance was used to determine vertical jump height changes according to pre-test and post-test measurements of the groups.
\end{abstract}

\section{Özet}

Giriş ve Amaç: Bu çalışmanın amacı baş üstü hedef göstererek yapılan dikey sıçrama egzersizlerinin voleybol oyuncularının dikey sıçrama performansina olan etkisini araştırmaktır. Yöntem: Çalışmaya on sekiz erkek voleybol (yaş: 21,3 $\pm 1,8$ y1l, boy:

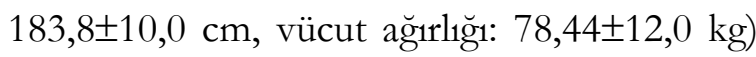
oyuncusu katılmıştır. Katılımcılar rastgele yöntemle iki gruba ayrılmıştır. Çalışma öncesi tüm kat1lımciların ön test olarak Sargent yöntemiyle dikey sıçrama yükseklikleri ölçülmüştür. Daha sonra haftada üç gün, toplam dört hafta boyunca standart antrenman sonrası kontrol grubu hedef gösterilmeden 10 dikey sıçrama egzersizi yaparken, çalışma grubu ön test sonucunun $\% 10$ fazlas1 hedef gösterilerek 10 sıçrama çalışması yapmışlardır. Gösterilen hedefe bir çalışmada 3 defa ulaşan bir katılımcının hedefi \%10 arttırılmıştır. Son test olarak her iki grubunda Sargent yöntemiyle maksimal sıçrama yükseklikleri tekrar ölçülmüştür. Grupların ön test ve son test ölçümlerine göre dikey sıçrama yükseklik değişimlerinin belirlenmesi için 2X2 tekrarlı ölçümler varyans analizi uygulanmıştır.

\footnotetext{
${ }^{1}$ Bu çalışma 5. Uluslararası Spor Bilimleri, Turizm ve Rekreasyon Öğrenci Kongresinde sözel bildiri olarak yayınlanmıştır.

2 Dr. Öğr. Üyesi, Afyon Kocatepe Üniversitesi, Beden Eğitimi ve Spor Yüksekokulu, mehmetyildiz@aku.edu.tr

${ }^{3}$ Afyon Kocatepe Üniversitesi, Beden Eğitimi ve Spor Yüksekokulu, engine oguzhan@,hotmail.com
} 
Yildiz, M., \& Engin, O. (2019). Hedef gösterilerek yapılan pliometrik çalıșmalar voleybolcuların dikey sıçrama performansını daha fazla geliştiriyor. Journal of Human Sciences, 16(4), 951-958. doi:10.14687/jhs.v16i4.5831

Results: During the study, the maximal jump values of the volleyball players were found to increase by $13 \%(55,67 \pm 4,56 \mathrm{~cm})$ compared to the control group $(50,56 \pm 7,70 \mathrm{~cm})$. Conclusion: In branches such as volleyball, where jump performance is the most important determinant of success, it is suggested that exercises should be performed through an overhead target to improve vertical jump performance.

Keywords: Volleyball, overhead target, jump, extrinsic motivation, plyometric.

(Extended English summary is at the end of this document)
Bulgular: Çalışma sırasında hedef göstererek $(55,67 \pm 4,56 \mathrm{~cm})$ yapilan egzersizlerin kontrol grubuna oranla $(50,56 \pm 7,70 \quad \mathrm{~cm})$ voleybolcularda maksimal sıçrama değerlerinin daha fazla arttığ1 (\%13) tespit edilmiştir. Tartışma-Sonuç: Voleybol gibi dikey sıçrama performansının başarının en önemli belirleyicisi olduğu branşlarda siçrama performansının geliştirilmesi için baş üstü hedef gösterilerek yapılacak egzersizler önerilmektedir.

Anahtar Kelimeler: Voleybol, baş üstü hedef, sıçrama, dişsal motivasyon, pliometrik.

\section{Giriş}

Dikey Siçrama hareketleri olan servis, set, smaç ve blok gibi birçok temel bir hareket voleybol performansının en önemli belirleyicisidir (Ziv ve Lidor, 2010). Dikey sıçramalar voleybol antrenmanları ve müsabakalarında hem savunmada hem de hücumda yoğun olarak kullanılmaktadır (Gladden ve Colacino, 1978). Elit erkek voleybolcular üzerine yapılan hareket analizi sonucuna göre, öndeki oyuncular adımlı olarak veya adım almadan maksimal yada maksimale yakın sıçrama performans1 göstermektedirler (Sheppard ve ark. 2009). Voleybolda takım başarısının belirlenmesinde, blok ve adım alarak sıçramalar en önemli becerilerdir (Lidor ve Ziv, 2010). Ayrıca, bu sıçrama hareketlerinin başarısı filenin üzerinde yapıldıkları yüksekliğe bağlıdır (Nejic ve ark., 2013). Daha iyi performans gösteren oyuncuların hem beceri, hem de dikey stçrama performansını beraber sergileyen oyuncular olduğu bildirilmiştir (Sattler ve ark., 2015; Smith ve ark, 1992).

Pliometrik veya balistik tipteki egzersizler dikey sıçrama performanslarını arttırmada sık kullanılan yöntemlerdir. Bu tür antrenmanlardan yüksek verim alınabilmesi için oyuncuların tam anlamıyla maksimal performans göstermesi gerekir. Çünkü güç veya güçteki en büyük artış maksimum yük kullanılarak elde edilir. Ancak, uzun süreli, tekrarlanan ve monoton antrenmanlar oyuncuların motivasyonunu azaltabilmektedir. $\mathrm{Bu}$ nedenle, güç-hız çalışmaları sırasında oyuncuların motivasyonunun yüksek tutulması için belirli koşullar yaratmak gerekmektedir. Genel olarak geri bildirim (Newton ve Kraemer, 1994) ve sözel cesaretlendirme içeren dişsal motivasyon (Andreacci ve ark., 2002; Moffatt ve ark.,, 1994; Chitwood ve ark., 1997; Işık ve ark., 2015) performans sonuçlarını geliştirmek için birçok çalışmada kullanılmıştır. Bununla beraber baş üstü hedef gösterilerek (dışsal geri bildirim) yapılan dikey sıçrama çalışmalarının voleybol oyuncularının dikey sıçrama performansı üzerine etkisini gösteren sınırlı sayıda bilgi bulunmaktadır. Bu çalışmanın amacı baş üstü hedef gösterilerek yapılan dikey sıçrama çalışmalarının voleybolcuların dikey sıçrama performansı üzerine etkisini araştırmaktır.

\section{Metot}

\section{Katılımcilar}

Çalışmaya üniversite erkek voleybol takımında (1. Lig) oynayan 18 sporcu oyuncusu ( yaş: $21,3 \pm 1,8$ yll, boy: $183,8 \pm 10,0$ cm, vücut ağırllğı: $78,44 \pm 12,0 \mathrm{~kg}$ ) gönüllü olarak katılmıştır. Katılımcılar en az 4 yil voleybol geçmişine sahip, son 6 aydır herhangi bir kas-iskelet sistemi yaralanması yaşamamış ve son iki ay/haftada 3gün/günde 1-2 saat arası düzenli antrenman yapan voleybolculardan seçilmiştir. Tüm katıllımcılar gönüllü olur formunu imzalamıştır. 
Yildiz, M., \& Engin, O. (2019). Hedef gösterilerek yapılan pliometrik çalıșmalar voleybolcuların dikey sıçrama performansını daha fazla geliştiriyor. Journal of Human Sciences, 16(4), 951-958. doi:10.14687/jhs.v16i4.5831

\section{Deneysel tasarım}

Baş üstü bir hedefinin dikey sıçrama yükseklik performansı üzerindeki etkisini belirlemek için katıllımcılar rastgele yöntemle iki gruba ayrılmıştır: (1) Baş üstü hedef grubu [BÜH grubu ( $\mathrm{n}=9$; yaş:

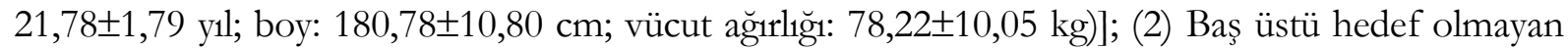

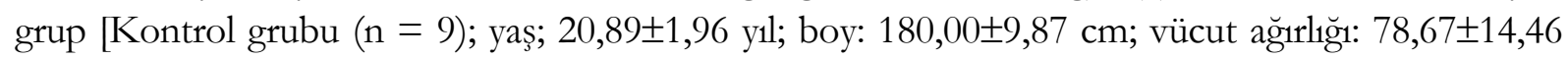
$\mathrm{kg})$ ]. Randomizasyon işlemi, www.randomizer.org web sitesi kullanılarak elektronik ortamda gerçekleştirilmiştir. BÜH grubu, düzenli antrenman programından sonra maksimal sıçrama yüksekliğinin \%10’u kadar artırllmış olan baş üstündeki yüksekliği hedefleyerek dikey sıçrama antrenmanı yaparken, kontrol grubu, normal antrenman programindan sonra herhangi bir hedef gösterilmeden dikey sıçrama çalışmaları gerçekleştirmişlerdir. Çalışma öncesi ve sonrası tüm katıllımcıların dikey sıçrama yükseklik performansları Sergant yöntemiyle analiz edilmiştir. Katılımcılar, haftada üç seans olmak üzere dört haftalık dikey sıçrama antrenmanı yapmışlardır. Çalışma sonunda gruplar arası dikey sıçrama yükseklik performansları karşılaştırılmıştır.

\section{Prosedür}

Katılımcıların laboratuvara geldiklerinde ilk olarak yaş, boy, ve vücut ağırlıkları tanımlayıc1 istatistikler olarak değerlendirilmiştir. Katılımcılar submaksimal koşu ve serbest dinamik gerdirme içeren 10 dakikalık bir ısınma egzersizinden sonra, Sıçra Eriş Cihazı (Sergant yöntemi) katılımcıların dikey pozisyonda kollarını kaldırarak parmak uçları cihazın en altında bulunan kayan parçaya değecek şekilde ayarlanmıştır. Her katılımcıya göre ayarlanmış olan cihazda katılımcının durduğu yerde eller salık pozisyonda maksimal sıçrama sonrası dokunduğu döner parçanın yüksekliği dikey sıçrama yüksekliği olacağı anlatılmıştır. İlk olarak araştırmacı tarafindan sıçrama şekli katulımcılara gösterilmiştir. Daha sonra her katılımcıya ayarlanmış şekli ile cihaz üzerinde katılımcıların 3 deneme yapması sağlanmıştır. 3 deneme içinde en yüksek değer ön-test sonucu olarak kaydedilmiştir.

BÜH grubunun ön-test sonuçlarının belirlenmesinden sonra, araştırmacı tarafindan gruptaki her katılımcı için en yüksek ön-test değeri $\% 10$ artırarak Sıçra Eriş cihazında hedef yükseklik belirlenmiştir. Bu hedefe göre katılımcılardan hedefe sıçramaları ve vurmaları istenmiştir. Bir antrenman seansında her katılımcı toplam 10 dikey sıçrama gerçekleştirmiştir. Katılımcının bir antrenman seansında hedefe üç kez vurması halinde, bir sonraki seansta hedefin yüksekliği \%10 oranında arttırılmıştır. Araştırmacı tarafindan sözlü cesaretlendirme ve maksimum sıçrama yükseklikleri hakkında bilgilendirici geri bildirim yapılmıştır. Sıçramalar arasında 30 saniyelik bir dinlenme aralığ verilmiştir. Kontrol grubu, bir antrenman seansında herhangi bir hedef gösterilmeden oldukları yerde toplam 10 dikey sıçrama gerçekleştirmiştir. Kontrol grubundaki katılımcılara geri bildirim olmadan sadece sözlü cesaretlendirme verilmiştir. Her iki grup da eller salık pozisyonda 30 sn dinlenme aralıkları ile dikey sıçramaları gerçekleştirmiş̧ir. Çalışma sonunda tüm katılımcıların dikey sıçrama yükseklik performansları ön-testte uygulanan aynı protokole göre yeniden ölçülmüsstür. Ölçümler aynı kapalı spor alanında ve 16:00-19:00 saatleri arasında yapılmıştır. Ölçümler öncesi katılımcılardan aynı diyet programını takip etmeleri, kafein ve alkol kullanmamaları istenmiştir.

\section{İstatistiksel analiz}

İstatistiksel analizler için SPSS 18.0 programı kullanılmıştır. Grup değerlerin ortalama \pm standart sapma değerleri gösterilmiştir. Verilerin normallik varsayımını analiz etmek için KolmogorovSmirnov testi yapılmıştır. Verilerin normal dağıldığı görüldükten sonra, gruplar arasındaki farkları belirlemek için bağımsız örneklem $t$ testi ve grup zaman etkileşiminin belirlenmesi için 2 (Grup) $\times 2$ (Zaman: ön-test, son-test) tekrarlı ölçümler ANOVA testi kullanılmıştır. Ölçümlerin güvenilirliği için sınıf içi korelasyon katsayısı testi kullanılmış ve değerlerin 0,80 ile 0,92 arasında olduğu tespit edilmiştir.

\section{Bulgular}


Yildiz, M., \& Engin, O. (2019). Hedef gösterilerek yapılan pliometrik çalıșmalar voleybolcuların dikey sıçrama performansını daha fazla geliştiriyor. Journal of Human Sciences, 16(4), 951-958. doi:10.14687/jhs.v16i4.5831

Tablo 1'de görüldüğü gibi, grup arasında yaş, boy ve vücut ağırlı̆̆ değişkenleri arasında anlamlı bir fark tespit edilememiştir.

Tablo 2'de görüleceği üzere, çalışma sonrası hedef göstererek $(55,67 \pm 4,56 \mathrm{~cm})$ yapılan egzersizlerin kontrol grubuna oranla $(50,56 \pm 7,70 \mathrm{~cm})$ voleybolcularda maksimal siçrama değerlerini daha fazla arttığ $(\% 13, \mathrm{P}<0,001)$ tespit edilmiştir

Tablo 1. Katılımcıların tanımlayıcı istatistiklerinin karşılaştırılması

\begin{tabular}{|c|c|c|}
\hline Değişkenler & Grup & $\bar{X} \pm \mathrm{sS}$ \\
\hline \multirow[b]{2}{*}{ Yaş (y1l) } & BÜH grubu & $21,78 \pm 1,79$ \\
\hline & Kontrol grubu & $20,89 \pm 1,96$ \\
\hline \multirow[b]{2}{*}{ Boy $(\mathrm{cm})$} & BÜH grubu & $180,78 \pm 10,80$ \\
\hline & Kontrol grubu & $180,00 \pm 9,87$ \\
\hline \multirow[b]{2}{*}{ Kilo (kg) } & BÜH grubu & $78,22 \pm 10,05$ \\
\hline & Kontrol grubu & $78,67 \pm 14,46$ \\
\hline
\end{tabular}

Tablo 2. BÜH grubu (n: 9) ve Kontrol grubunun (n: 9) ön test ve son test ölçümlerine göre sıçrama yükseklik değişimlerini gösteren tekrarlı ölçümler varyans analizi tablosu

\begin{tabular}{|c|c|c|c|c|c|}
\hline & $\begin{array}{l}\text { Ön-test } \\
\bar{X} \pm \mathrm{sS}\end{array}$ & $\begin{array}{l}\text { Son-test } \\
\bar{X} \pm \mathrm{SS}\end{array}$ & $\begin{array}{c}\text { Total } \\
\bar{X} \pm \mathrm{SS}\end{array}$ & $\mathrm{f}$ & $\mathrm{p}$ \\
\hline BÜH grubu & $49,00 \pm 6,75$ & $55,67 \pm 4,56$ & $52,33 \pm 2,27$ & \multirow{2}{*}{82,296} & \multirow{2}{*}{0,000} \\
\hline Kontrol grubu & $47,33 \pm 8,03$ & $50,56 \pm 7,70$ & $48,94 \pm 2,27$ & & \\
\hline Total & $48,17 \pm 7,25$ & $53,11 \pm 6,68$ & & & \\
\hline \multicolumn{6}{|c|}{$f: 1,119 ; p: 0,336$} \\
\hline
\end{tabular}

Grup x zaman etkileşimi: f: 9,984; p: 0,06

\section{Tartışma}

Bu çalışmanın amacı hedef gösterilerek yapılan dikey sıçrama çalışmalarının voleybolcuların dikey sıçrama performansı üzerine etkisini araştırmaktır. Çalışma öncesi tüm katılımcıların ön test olarak Sargent yöntemiyle sıçrama yükseklikleri ölçülmüştür. Kontrol grubu hedef gösterilmeden durarak 10 sıçrama egzersizi yaparken, çalışma grubu ön test sonucunun \%10 fazlası hedef gösterilerek 10 sıçrama çalışması yapmışlardır. Çalışma haftada üç gün, toplam dört hafta sürmüştür. Son test olarak her iki grubunda Sargent yöntemiyle maksimal sıçrama yükseklikleri tekrar ölçülmüştür. Çalışma sirasinda hedef göstererek $(55,67 \pm 4,56 \mathrm{~cm})$ yapilan egzersizlerin kontrol grubuna oranla $(50,56 \pm 7,70$ $\mathrm{cm})$ voleybolcularda maksimal sıçrama değerlerini daha fazla arttı̆̆ $(\% 13)$ tespit edilmiştir.

Bulgularımız, uzun dönem dişsal motivasyonun sıçrama performansı üzerindeki etkilerini inceleyen önceki araştırmalarla paralellik göstermektedir (Makaruk ve ark. 2012; Keller ve ark., 2014). Makaruk ve ark. (2012) 36 fiziksel olarak aktif erkek katılımcıyla dikkatin odaklamasının sıçrama performansı üzerine etkisini araştırdığı çalışmasında, dışsal motivasyonun içsel motivasyon ve kontrol grubuna göre daha fazla gelişim sağladığını bildirmiştir. Benzer şekilde, Keller ve ark. (2014) 4 haftalık bir geri bildirim yapılan pliometrik antrenmandan sonra (uzun süreli) geri bildirim yapılmayan çalışmalara göre hem akut hem de uzun dönemli olarak sıçrama yüksekliğini daha fazla geliştirdiğini bildirmiştir (Keller ve ark., 2014). Bunun yanında, çok sayıda araştırma dışsal motivasyonun akut olarak derinlik sıçrama (Mok ve ark., 2017; Ford ve ark., 2005), aktif dikey siçrama (Krol ve ark., 2016; Wulf ve ark., 2007; Wulf ve Dufek, 2009) ve durarak uzun atlama (Porter ve aerk., 2010) performansını geliştirdiğini göstermektedir. Krol ve ark. (2016), hedef olmayan çalışmalara kıyasla, hedef gösterilerek yapılan aktif dikey sıçramalarında daha yüksek performans belirlemişlerdir. Bir 
Yildiz, M., \& Engin, O. (2019). Hedef gösterilerek yapılan pliometrik çalıșmalar voleybolcuların dikey sıçrama performansını daha fazla geliştiriyor. Journal of Human Sciences, 16(4), 951-958. doi:10.14687/jhs.v16i4.5831

diğer çalışmada, Mok ve ark. (2017), baş üstünde gösterilen bir hedefin, hedef gösterilmeden yapılan derinlik sıçramalarına oranla daha iyi sıçrama yüksekliği performansı gösterdiğini bildirmişlerdir.

Ford ve ark., (2017) sanal olarak gösterilen baş üstü bir hedef ile fiziksel bir baş üstü hedefin dikey sıçrama yükseklik performansı ve sıçma biyomekaniği üzerindeki etkilerini araştırdıkları çalışmalarında hem fiziki hedefin, hem de sanal hedeflerinin, derinlik sıçrama sırasındaki sıçrama yüksekliğini arttırdığını tespit etmişlerdir. Ayrıca, bazı araştırmacılar maksimal kuvvet ölçümlerinde geri bildirim almayan katılımcılarla karşılaştırlldı̆ında katılımcılar artırılmış geri bildirim aldıktan sonra daha iyi kuvvet/tork ortaya çıkardıklarını göstermişlerdir (Figoni ve Morris, 1984; Hopper ve ark., 2003, Peacock ve ark., 1981). Ayrıca, çok sayıda çalışma dişsal bir motivasyonun (sözel teşviklerin) maksimum test sonuçlarını geliştirdiğini tespit etmiştir (Andreaccı ve ark., 2002; Moffatt ve ark, 1994; Chitwood ve ark, 1997). Bu nedenle, maksimal testlerin etkinliğini arttırmak için dışsal bir motivasyonun kullanılması gerektiği öne sürülmüştür (Newton ve Kraemer, 1994). Newton ve Kraemer (1994) geri bildirim veya genel amaçlı bir hedefler kullanmanın güç üretimini optimize edebileceğini ileri sürmüştür.

Baş üstü hedef kullanılarak yapılan pliometrik çalışmalardaki performans gelişimi, dışsal dikkatin odaklanması ve motorik işlemlerin otomatik kontrol süreçleriyle kendi kendine organize edilmesine izin verdiği kısıtlı eylem hipotezi ile açıklanabilir. Ayrıca, bazı çalışmalarda sıçrama yüksekliği performans kazanımlarının olası açıklaması motivasyonel faktörlere atfedilmiştir (Keller ve ark., 2014; Moran ve ark., 2012). Sporcuların maksimal veya o esnadaki performanslan hakkındaki geri bildirimleri motivasyonlarını artırabilir ve ellerinden gelenin en iyisini göstermesine yol açabilir. Buna ek olarak, bazı araştırmacılar dışsal dikkatin yoğunlaştırılması ile daha fazla kas lifinin kasılmaya işlemine katıldığı ve daha etkili hareket düzeni oluştuğunu ve bu durumun performans gelişsimini sağladığını bildirmiştir (Fort ve ark, 2017; Horita ve ark., 2002). Şimdiki çalışmada, deney grubunda dışsal odaklanma için baş üstü bir hedef kullanılmıştır. Ayrıca, katılımcılara sıçrama performansları hakkında anında geri bildirim yapılmıştır. $\mathrm{Bu}$ nedenle, $\mathrm{BÜH}$ grubunda görülen sıçrama yüksekliğindeki artş literatüre paralel olarak gelişmiş bir hareket düzeni ve daha fazla kas lifinin kasılmaya katılmasının sonucu olarak açıklanabilir.

\section{Sonuç ve Öneriler}

Bu çalışmanın amacı hedef gösterilerek yapılan dikey sıçrama çalışmalarının voleybolcuların dikey sıçrama performansı üzerine etkisini araştırmaktır. Çalışma sırasında hedef göstererek yapılan pliometrik egzersizlerin hedef gösterilmeden yapılan çalışmalara oranla voleybolcularda maksimal sıçrama değerlerini daha fazla arttığı tespit edilmiştir. Voleybolda takım başarısının belirlenmesinde, dikey sıçrama performansı en önemli becerilerden biridir. Ayrıca, bu sıçramaların etkinliği, ağın üzerinde yapıldıkları yüksekliğe bağlıdır. Bununla birlikte, bu tip hareketler güç-hız eylemleridir ve performans alt ekstremitelerin ekstansiyonu sırasında büyük miktarda dış kuvvetin üretimine bağlıdır. Kuvvet-hız hareketlerindeki optimal performans artısıs vücuda binen yükle orantılıdır ve bunun için sporcunun iyi bir uyarılmışlık ve bilinç düzeyinde olması gerekir. Bununla birlikte uzun süreli ve monoton antrenmanlar sporcularnn motivasyon seviyesini azaltabilmektedir. Bu nedenle, sporcuların motor görevleri tam olarak yerine getirilmesi için eğitmenler veya profesyoneller tarafindan özel koşullar yaratılmalıdır. Buna göre Antrenör ve çalıştırıcılar voleybol oyuncularının dikey sıçrama yüksekliklerini arttırmak için baş üstü hedefleri dışsal motivatör olarak kullanarak performans gelişimini sağlayabilirler.

\section{Kaynakça}


Yildiz, M., \& Engin, O. (2019). Hedef gösterilerek yapılan pliometrik çalıșmalar voleybolcuların dikey sıçrama performansını daha fazla geliştiriyor. Journal of Human Sciences, 16(4), 951-958. doi:10.14687/jhs.v16i4.5831

Andreacci, J. L., Lemura, L. M., Cohen, S. L., Urbansky, E. A., Chelland, S. A., \& Duvillard, S. P. V. (2002). The effects of frequency of encouragement on performance during maximal exercise testing. Journal of sports sciences, 20(4), 345-352.

Chitwood, L., Mop att, R., Burke, K., Luchino, P. and Jordan, J. (1997). Encouragement during maximal exercise testing of Type A and Type B scorers. Perceptual and Motor Skills, 84, $507 \pm 512$.

Cohen J. Statistical Power Analysis for the Behavioral Sciences. Hillsdale, N.J. : Lawrence Erlbaum, 1988.

Figoni, S. F., \& Morris, A. F. (1984). Effects of knowledge of results on reciprocal, isokinetic strength and fatigue. Journal of Orthopaedic \& Sports Physical Therapy, 6(3), 190-197.

Ford, K. R., Myer, G. D., Smith, R. L., Byrnes, R. N., Dopirak, S. E., \& Hewett, T. E. (2005). Use of an overhead goal alters vertical jump performance and biomechanics. The Journal of Strength \& Conditioning Research, 19(2), 394-399

Ford, K. R., Nguyen, A. D., Hegedus, E. J., \& Taylor, J. B. (2017). Vertical Jump Biomechanics Altered With Virtual Overhead Goal. Journal of applied biomechanics, 33(2), 153-159.

Gladden, L. B., \& Colacino, D. (1978). Characteristics of volleyball players and success in a national tournament. The Journal of sports medicine and physical fitness, 18(1), 57.

Hopper, D. M., Berg, M. A. A., Andersen, H., \& Madan, R. (2003). The influence of visual feedback on power during leg press on elite women field hockey players. Physical Therapy in Sport, 4(4), 182-186.

Horita, T., Komi, P., Nicol, C., \& Kyröläinen, H. (2002). Interaction between pre-landing activities and stiffness regulation of the knee joint musculoskeletal system in the drop jump: implications to performance. European journal of applied physiology, 88(1-2), 76-84.

Işık, Ö., Ersöz, Y., Pazan, M., \& Ocak, Y. (2015). The effect of motivational music on wingate anaerobic test performance. Journal of Human Sciences, 12(2), 513-520.

Keller, M., Lauber, B., Gehring, D., Leukel, C., \& Taube, W. (2014). Jump performance and augmented feedback: immediate benefits and long-term training effects. Human movement science, 36, 177-189.

Krol, h., borzucka, d., \& rektor, z. (2016). Effect of an extrinsic motivator on mechanical parameters of the counter movement jump used in team sports. Jkes 73 (26): 49-58.

Lidor, R., \& Ziv, G. (2010). Physical and physiological attributes of female volleyball players-a review. The Journal of Strength \& Conditioning Research, 24(7): 1963-1973.

Moffatt, R. J., Chitwood, L. F., \& Biggerstaff, K. D. (1994). The influence of verbal encouragement during assessment of maximal oxygen uptake. The Journal of sports medicine and physical fitness, 34(1), 45-49.

Mok, K. M., Bahr, R., \& Krosshaug, T. (2017). The effect of overhead target on the lower limb biomechanics during a vertical drop jump test in elite female athletes. Scandinavian journal of medicine \& science in sports, 27(2), 161-166.

Moran, K. A., Murphy, C., \& Marshall, B. (2012). The need and benefit of augmented feedback on service speed in tennis. Medicine \& Science in Sports \& Exercise, 44(4), 754-760.

Newton, R. U., \& Kraemer, W. J. (1994). Developing explosive muscular power: Implications for a mixed methods training strategy. Strength \& Conditioning Journal, 16(5), 20-31.

Nejic, D., Trajković, N., Stanković, R., Milanović, Z., \& Sporiš, G. (2013). A comparison of the jumping performance of female junior volleyball players in terms of their playing positions. Facta Universitatis. Series: physical dducation and sport, 11(2), 157-164.

Peacock, B., Westers, T., Walsh, S., \& Nicholson, K. (1981). Feedback and maximum voluntary contraction. Ergonomics, 24(3), 223-228.

Porter, J. M., Ostrowski, E. J., Nolan, R. P., \& Wu, W. F. (2010). Standing long-jump performance is enhanced when using an external focus of attention. The Journal of Strength \& Conditioning Research, 24(7), 1746-1750. 
Yildiz, M., \& Engin, O. (2019). Hedef gösterilerek yapılan pliometrik çalıșmalar voleybolcuların dikey sıçrama performansını daha fazla geliştiriyor. Journal of Human Sciences, 16(4), 951-958. doi:10.14687/jhs.v16i4.5831

Sheppard, J. M., Gabbett, T. J., \& Stanganelli, L. C. R. (2009). An analysis of playing positions in elite men's volleyball: considerations for competition demands and physiologic characteristics. The Journal of Strength \& Conditioning Research, 23(6), 1858-1866.

Smith, D. J., Roberts, D., \& Watson, B. (1992). Physical, physiological and performance differences between Canadian national team and universiade volleyball players. Journal of sports sciences, 10(2), 131-138.

Wulf, G., \& Dufek, J. S. (2009). Increased jump height with an external focus due to enhanced lower extremity joint kinetics. Journal of motor behavior, 41(5), 401-409.

Wulf, G., Zachry, T., Granados, C., \& Dufek, J. S. (2007). Increases in jump-and-reach height through an external focus of attention. International Journal of Sports Science \& Coaching, 2(3), 275284

Ziv, G., \& Lidor, R. (2010). Vertical jump in female and male volleyball players: a review of observational and experimental studies. Scandinavian journal of medicine \& science in sports, 20(4), 556-567.

\section{Extended English Summary}

Vertical jumps used in both defense and attacks in volleyball are among the most important determinants of performance. Plyometric and ballistic exercises are the most important methods to improve vertical jump performance. In order to get high efficiency from this kind of training, players need to show maximum performance. However, a long-term, repeated and monotonous training reduces the motivation of the players. External motivation (Andreacci et al., 2002; Moffatt et al., 1994; Chitwood et al., 1997), which generally includes feedback (Newton and Kraemer, 1994) and verbal encouragement, has been used in many studies to improve performance outcomes. However, there is a limited amount of information showing the effect of overhead target (vertical feedback) on volleyball players' vertical jump performance. The purpose of this study was to investigate the effect of jumping exercises with a target on the jumping performance.

Eighteen male volleyball players (age $21.3 \pm 1.8$ years, height $183.8 \pm 10.0 \mathrm{~cm}$, weight 78.44 $\pm 12.0 \mathrm{~kg}$ ) playing in the university volleyball team (1st Division) participated in the study voluntarily. The participants were selected from volleyball players who had at least four years of volleyball history, had not suffered from any musculoskeletal injuries in the last three months and who had regularly trained 1-2 hours a day for three days in a week. All participants signed the voluntary participation form.

Before the study, as a pre-test, jumping heights of all participants were measured using the Sargent method. Then the participants were divided into two groups as the Overhead Target group (OHTG) and Control group (CG) randomly. After measuring pretest of groups, the CG performed 10 vertical jumps without a target and the OHTG performed 10 jumps with a overhead target in which the distance of the overhead target was more than $10 \%$ of the pre-test results. The study included 12 training session (four weeks/ 3days in a week).

When a participant in the OHTG hit the target three times during a vertical jump in one training session, the height of the target was increased by $10 \%$ in the next session. The researcher gave verbal encouragement and informative feedback on maximum jump heights. A rest interval of 30 seconds was given between the jumps. The participants in the CG were given only oral encouragement without feedback. At the end of the study, vertical jump height performances of all participants were re-measured according to the same protocol applied in the pre-test. Measurements were conducted in the same indoor sports area between 16:00-19: 00. Before the measurements, the participants were asked to follow the same diet program and not to use caffeine and alcohol before tests and training sessions. SPSS 18.0 program was used for statistical analysis. 
Yildiz, M., \& Engin, O. (2019). Hedef gösterilerek yapılan pliometrik çalışmalar voleybolcuların dikey sıçrama performansını daha fazla geliştiriyor. Journal of Human Sciences, 16(4), 951-958. doi:10.14687/jhs.v16i4.5831

Mean \pm standard deviation of group values was shown. Kolmogorov-Smirnov test was used to analyze the assumption of normality of data. After the normal distribution of the data, independent sample $t$ test was used to determine the differences between the groups for pre-test and post-test results, and dependent variable t test was used to determine intra-group differences. Moreover, repeated measurements ANOVA test was used to determine group time interaction $2 \times 2$ (Time: pre-test, post-test) repeated measurements. During the study, the maximal jump values of the OHTG were found to increase by $13 \%(55,67 \pm 4,56 \mathrm{~cm})$ when compared with the CG $(50,56 \pm$ $7,70 \mathrm{~cm})$.

Our findings have been in accordance with previous studies examining the effects of longterm extrinsic motivation on jump performance (Makaruk et al. 2012; Keller et al., 2014). Makaruk et al. (2012) investigated the effect of attention focus on jumping performance, and reported that extrinsic motivation provided more improvement compared intrinsic motivation. Additionlly, many studies have shown that external motivation increased drop jump (Mok et al., 2017; Ford et al., 2005), counter movement jump (Krol et al., 2016; Wulf et al., 2007; Wulf and Dufek, 2009), and standing long jump performance (Porter et al., 2010) acutely.

The aim of this study was to investigate the effect of an overhead target on volleyball players' vertical jump performance. It was found that vertical jump exercises performed using an overhead target increased the maximal jump values of the volleyball players more than the without targets exercises. Vertical jump performance is one of the most important skills in determining team success in volleyball. However, such movements are power-speed actions and performance depends on the generation of a large amount of external force during the extension of the lower extremities. Therefore, trainers and coaches can improve performance using an overhead targets as external motivators to increase the vertical jump height of volleyball players. 